\title{
Electrophysiological biomarkers for improved etiological diagnosis of cognitive impairment
}

This article was published in the following Dove Press journal:

Current Biomarker Findings

7 July 2014

Number of times this article has been viewed

\author{
Takao Yamasaki ${ }^{1,2}$ \\ Shozo Tobimatsu' \\ 'Department of Clinical \\ Neurophysiology, Neurological \\ Institute, Graduate School of \\ Medical Sciences, Kyushu University, \\ Fukuoka, Japan; ${ }^{2}$ Department of \\ Neurology, Minkodo Minohara \\ Hospital, Fukuoka, Japan
}

\begin{abstract}
Alzheimer's disease (AD), Lewy body disease (LBD), and frontotemporal lobar degeneration (FTLD) are three major types of neurodegenerative dementia. Intervention and treatment differ significantly among these major dementias, necessitating early and accurate diagnosis. Patients with AD, LBD, and FTLD exhibit specific patterns of visual dysfunction as early behavioral signs. These visual impairments are the manifestations of topographic patterns of neuropathology specific to each type of dementia. Electrophysiological measurements, such as visual evoked potentials and event-related potentials, are objective and noninvasive tools that can detect subtle functional changes in human brain. Therefore, with the use of visual evoked potentials and event-related potentials, early detection of specific patterns of visual dysfunction may be useful for differential diagnosis of dementia. In this review, we first summarize current knowledge about the relevant aspects of the human visual system. Second, we outline clinical characteristics, including visual perceptual abnormalities, of each type of degenerative dementia. Finally, we describe the application of visual evoked potential and event-related potential recording techniques to study visual perception in patients with mild cognitive impairment (prodromal stage of dementia). We stress that electrophysiological signals have potential as reliable biomarkers in the diagnosis of different types of dementia, especially in the case of overlapping phenotypes.
\end{abstract}

Keywords: Alzheimer's disease, Lewy body disease, frontotemporal lobar degeneration, visual evoked potentials, event-related potentials, parallel visual pathways

\section{Introduction}

Progressive cognitive impairment (or dementia) is one of the most serious conditions associated with longevity, and represents a pressing public health problem. There are many different types of dementia, most of which are irreversible and degenerative. ${ }^{1}$ Alzheimer's disease (AD), Lewy body disease (LBD), and frontotemporal lobar degeneration (FTLD), which each have different underlying etiologies and pathogenic mechanisms, are the three main types of neurodegenerative dementia. ${ }^{2-4}$ $\mathrm{AD}$ is the most common and most studied cause of dementia. ${ }^{5} \mathrm{~A}$ large number of novel compounds with potential for modifying the course of AD and slowing its progression are currently in development. Thus, early and accurate diagnosis of the specific dementia type (in particular, AD) has become increasingly important. The diagnosis of degenerative dementias remains difficult because of the heterogeneity of the types and the partial overlap of their clinical presentations. ${ }^{1,4}$ For this reason, sensitive and specific biomarkers for early and differential diagnosis of dementia are urgently needed.
Department of Clinical Neurophysiology, Neurological Institute, Graduate School of Medical Sciences, Kyushu University, 3-I-I Maidashi, Higashi-ku,

Fukuoka 8| 2-8582, Japan

Tel +81926425542

Fax +819 26425545

Email yamasa@neurophy.med.kyushu-u. ac.jp 
Neuropsychological, neurophysiological, biological, and genetic biomarkers have been proposed for the diagnosis of dementia. ${ }^{6}$ Electrophysiological activity is a type of neurophysiological biomarker, and electroencephalography, evoked potentials, and event-related potentials (ERPs) are the most common electrophysiological methods. ${ }^{7,8}$ These measurements, which are recorded from the scalp, are objective and noninvasive, and can be used to detect subtle functional changes that are not evident at clinical or behavioral levels. These techniques also provide a direct and real-time index of neuronal activity, with a resolution on the scale of milliseconds, and are relatively easy and inexpensive to implement. ${ }^{7.8}$ Therefore, electrophysiological measures are ideally suited to addressing differences in cognitive processing speed between normal individuals and individuals with dementia (particularly, prodromal stage of dementia), and between dementia subtypes.

Different types of neurodegenerative dementia, including $\mathrm{AD}, \mathrm{LBD}$, and FTLD, exhibit unique patterns of visual dysfunction as early behavioral signs ${ }^{9}$ (see later on Characteristics of degenerative dementia). These disease-related visual impairments are manifestations of changing topographical patterns of cortical activity. ${ }^{9}$ Accordingly, visual evoked potentials (VEPs) and ERPs may be sensitive tools in predicting pathology, for instance, in discriminating $\mathrm{AD}$ from other types of degenerative dementia. Electroretinography may also be useful in the differential diagnosis of dementia, as several reports have described a relationship between anterior visual pathway pathology, including retinal dysfunction, and the major neurodegenerative dementias. ${ }^{10-12}$ However, in this review, we focus on the differential diagnosis between $\mathrm{AD}$ and other types of neurodegenerative dementia, such as LBD and FTLD, with an emphasis on the relationship between cortical neuropathology and visual function.

In the present review, we first summarize current findings regarding the parallel visual pathways in humans. Second, we outline the clinical characteristics of each type of degenerative dementia, as well as the link between visual perceptual abnormalities and the human visual system. Finally, we present findings from a recent study in which we used VEPs and ERPs to examine visual perception in individuals with mild cognitive impairment (MCI, prodromal stage of dementia), and discuss the implications of this work. The main purpose of this paper is to illustrate how electrophysiological measurements associated with visual function can offer valuable quantitative biological markers for improving the differential diagnosis of major types of degenerative dementia.

\section{Human visual system}

In humans, visual processing is characterized by hierarchical and segregated processing. The parvocellular pathway and magnocellular pathway are two major parallel visual pathways. ${ }^{13}$ Both systems originate in the retina and project to the primary visual cortex via the lateral geniculate nucleus (lower-level visual pathway). After traveling to the primary visual cortex (higherlevel visual pathway), the parvocellular pathway projects to the ventral stream (responsible for processing "what" information), which includes the quaternary cortex and the inferior temporal cortex. The parvocellular pathway appears to be responsible for processing information about form and color because this system is characterized by high spatial resolution and color sensitivity, but low contrast sensitivity and temporal resolution. Conversely, after traveling to the primary visual cortex, the magnocellular pathway projects to the dorsal stream (responsible for processing "where" information), which includes V3A, V5/middle temporal area, medial superior temporal area, V6, and the posterior parietal lobule. The magnocellular pathway appears to play an important role in detecting motion because this system exhibits the opposite characteristics of the parvocellular pathway, ie, low spatial resolution and color insensitivity, but high contrast sensitivity and temporal resolution. The dorsal stream is also divided into two functional streams, ie, the dorsodorsal and ventrodorsal pathways. The former includes V6 and the superior parietal lobule, while the latter consists of the V5/middle temporal area and the inferior parietal lobule (Figure 1). For a review, see Tobimatsu and Celesia ${ }^{7}$ or Yamasaki and Tobimatsu. ${ }^{8}$

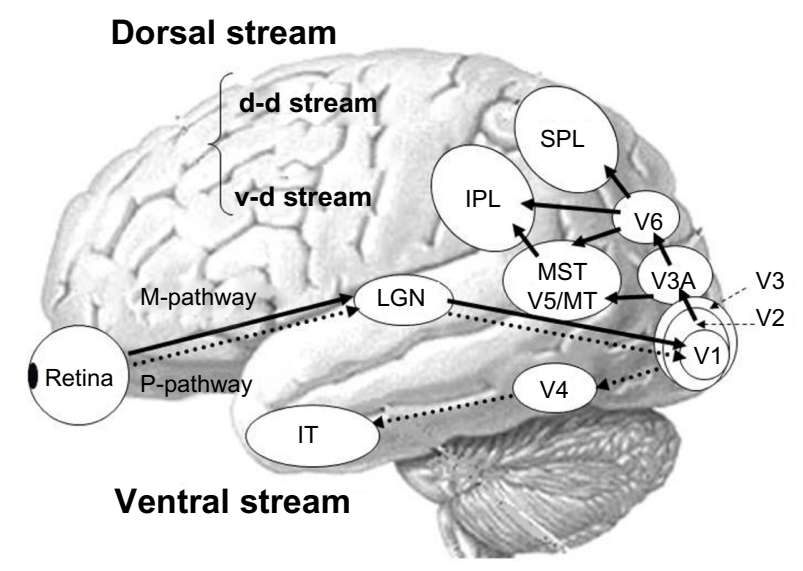

Figure I Schematic representation of the parallel visual pathways in humans. Note: Copyright (c) 2012 Takao Yamasaki et al. Adapted from Yamasaki T, Muranaka H, Kaseda Y, Mimori Y, Tobimatsu S. Understanding the pathophysiology of Alzheimer's disease and mild cognitive impairment: a mini review on $\mathrm{FMRI}$ and ERP studies. Neurol Res Int. 2012;2012:719056. ${ }^{49}$

Abbreviations: $M$, magnocellular; $P$, parvocellular; $d-d$, dorsodorsal; v-d, ventrodorsal; LGN, lateral geniculate nucleus; VI, 2, 3, 4 and 6, primary, secondary, tertiary, quaternary, and senary visual cortices; V3A, V3 accessory; V5/MT, quinary visual cortex/middle temporal area; MST, medial superior temporal area; IPL, inferior parietal lobule; SPL, superior parietal lobule; IT, inferior temporal cortex. 


\section{Characteristics of degenerative dementia}

The characteristics of the major types of degenerative dementia are summarized in Table 1.

\section{Alzheimer's disease}

$\mathrm{AD}$ is a progressive neurodegenerative disease, and the most common form of dementia in elderly people. ${ }^{5} \mathrm{AD}$ is characterized by impaired short-term episodic memory, orientation, visuospatial function, language, and executive function, but preserved motor function. ${ }^{1}$ The neuropathological hallmarks of $\mathrm{AD}$ include beta amyloid deposition in the form of senile plaques and accumulation of neurofibrillary tangles, which induce a series of toxic events that result in synaptic dysfunction, neuronal loss, and brain atrophy. ${ }^{14}$

Deficits in vision are common in people with AD. One neuropsychological study found that $43 \%$ of patients with AD had complex visual complaints. ${ }^{15} \mathrm{AD}$ patients also show impaired dorsal stream function, such as compromised angle discrimination and motion perception, and impaired ventral stream function, such as abnormal perceptual discrimination and recognition of faces, words, objects, and colors. ${ }^{16}$ In AD patients, progressive neuropathological changes, such as neuronal death, neurofibrillary tangles, and senile plaques, affect certain lamina and cell types within the neocortex. This may lead to corticocortical disconnection. The pathology of $\mathrm{AD}$ preferentially involves temporoparietal association areas, including the higher-level visual pathways. Conversely, the primary visual, motor, and somatosensory cortices are typically spared until very late stages of the disease., ${ }^{4,16}$ The pathological changes associated with $\mathrm{AD}$ indicate that the main source of visual impairment in $\mathrm{AD}$ patients is the degeneration of cells in the higher-level visual pathways (for a review, see Yamasaki et al). ${ }^{16}$

Posterior cortical atrophy is a clinical syndrome associated with prominent bottom-up visuospatial impairments with relative preservation of memory, insight, and judgment. ${ }^{17}$ Posterior cortical atrophy is associated with atrophy in the occipital, parietal, and posterior temporal lobes. Pathologically, posterior cortical atrophy is thought of as a form of $\mathrm{AD}$ with a greater frequency of neurofibrillary tangles in the visual cortex and a lower frequency of neurofibrillary tangles in the hippocampus. ${ }^{9}$ In comparison with typical $\mathrm{AD}$, patients with posterior cortical atrophy show selective hypometabolism of the occipitoparietal regions, with greater impairment in the right hemisphere compared with the left. ${ }^{18}$ The most common clinical features are dorsal stream (occipitoparietal) abnormalities, as well as several features of Balint's syndrome, such as simultanagnosia and optic ataxia. ${ }^{9}$ Indicators of ventral stream function, such as recognition of objects, faces, and colors, tend to be less frequently impacted than indicators of dorsal stream function. ${ }^{9}$ Accordingly, higher-level visual dysfunction (specifically higher-level dorsal dysfunction) with preserved function of the primary visual cortex is a characteristic feature of $\mathrm{AD}$.

Table I Characteristics of major types of degenerative dementia

\begin{tabular}{|c|c|c|c|c|}
\hline Dementia & Pathology & Clinical characteristics & Visual symptoms & Neuroimaging \\
\hline $\mathrm{AD}$ & $\begin{array}{l}\text { - Senile plaques } \\
\text { - Neurofibrillary } \\
\text { tangles }\end{array}$ & $\begin{array}{l}\text { - Beginning with memory } \\
\text { impairment and disorientation } \\
\text { - Spreading to language and } \\
\text { visuospatial deficits }\end{array}$ & $\begin{array}{l}\text { - Impaired recognition of faces, } \\
\text { objects, words, and colors } \\
\text { - Impaired perception of motion } \\
\text { and stereopsis (ventral and } \\
\text { dorsal impairments at higher- } \\
\text { level [dorsal impairments are } \\
\text { more severe, particularly in PCA]) }\end{array}$ & $\begin{array}{l}\text { - Hippocampal atrophy } \\
\text { - Diffuse or posteriorly predominant } \\
\text { cortical atrophy } \\
\text { - Posterior temporoparietal } \\
\text { hypoperfusion or hypometabolism } \\
\text { - Spares primary visual cortex }\end{array}$ \\
\hline LBD & $\begin{array}{l}\text { - } \alpha \text {-synuclein } \\
\text { inclusions (Lewy } \\
\text { bodies) }\end{array}$ & $\begin{array}{l}\text { - Early appearance of } \\
\text { parkinsonism, visual } \\
\text { hallucinations, fluctuating } \\
\text { cognitive impairment, or } \\
\text { rapid eye movement sleep } \\
\text { disorder }\end{array}$ & $\begin{array}{l}\text { - Impaired recognition of faces, } \\
\text { objects, and words } \\
\text { - Impaired perception of motion } \\
\text { and stereopsis (more severe } \\
\text { compared with AD) (ventral } \\
\text { and dorsal impairments at } \\
\text { both lower and higher levels) }\end{array}$ & $\begin{array}{l}\text { - Less hippocampal atrophy than AD } \\
\text { - Posterior temporoparietal } \\
\text { hypoperfusion or hypometabolism } \\
\text { - Occipital lobe (primary visual } \\
\text { cortex) hypoperfusion or } \\
\text { hypometabolism }\end{array}$ \\
\hline FTLD & $\begin{array}{l}\text { - Tau inclusions } \\
\text { - Pick bodies }\end{array}$ & $\begin{array}{l}\text { - Changes in personality and } \\
\text { behavior } \\
\text { - Speech and language deficits } \\
\text { - Multimodal agnosia } \\
\text { - Relatively spares memory } \\
\text { and visuospatial skills }\end{array}$ & $\begin{array}{l}\text { Losing the ability to recognize } \\
\text { faces (facial emotion), objects, } \\
\text { and words (ventral impairment } \\
\text { at higher levels) }\end{array}$ & $\begin{array}{l}\text { - Focal frontal and/or anterior } \\
\text { temporal atrophy } \\
\text { - Frontotemporal hypoperfusion or } \\
\text { hypometabolism } \\
\text { - Spares posterior parietal lobe }\end{array}$ \\
\hline
\end{tabular}

Abbreviations: AD, Alzheimer's disease; LBD, Lewy body disease; FTLD, frontotemporal lobar degeneration; PCA, posterior cortical atrophy. 


\section{Lewy body disease}

LBD is the second most common cause of neurodegenerative dementia, after $\mathrm{AD} .{ }^{19} \mathrm{LBD}$ is clinically characterized by dementia, parkinsonism, fluctuating cognitive impairment, attentional disturbances, visual hallucinations, and rapid eye movement sleep behavior disorder. ${ }^{19}$ LBD includes two clinical syndromes, dementia with Lewy bodies and Parkinson's disease with dementia. Dementia with Lewy bodies is diagnosed when dementia occurs before or concurrently with parkinsonism, while Parkinson's disease with dementia is characterized by the onset of dementia 12 months after the onset of parkinsonism..$^{20}$ In both dementia with Lewy bodies and Parkinson's disease with dementia, a characteristic neuropathological indicator is the presence of intraneuronal proteinaceous inclusions (Lewy bodies) primarily composed of $\alpha$-synuclein. ${ }^{20}$ One neuropathological difference between dementia with Lewy bodies and Parkinson's disease with dementia is the increased frequency of a coassociation with AD pathology in dementia with Lewy bodies. This may contribute to the rapid progression of dementia in individuals suffering from dementia with Lewy bodies, relative to the onset of motor symptoms. ${ }^{9}$

LBD patients exhibit visual impairments that are more severe and pervasive than those observed in AD patients. ${ }^{9}$ For example, patients with LBD (dementia with Lewy bodies or Parkinson's disease with dementia) were found to have more severe impairments than $\mathrm{AD}$ patients in tests of both ventral stream (tests of object and form perception) and dorsal stream (test of motion perception) function. ${ }^{9,21,22}$ These neuropsychological findings suggest that posterior cortical dysfunction is more severe in LBD than in AD. In neuroimaging studies, both LBD and $A D$ patients show hypoperfusion and hypometabolism in the parietal and temporal lobes, but only patients with dementia with Lewy bodies exhibit hypoperfusion or hypometabolism in the occipital lobes, including the primary visual cortex. ${ }^{9,23,24}$ The primary visual cortex is the most critical cortical region for early aspects of bottom-up visual processing. Thus, the results of these neuroimaging studies are consistent with neuropsychological findings. Accordingly, it seems that LBD patients have impairments that are related to both the ventral and dorsal streams, and affect the primary visual cortex as well as higher visual levels. In particular, impaired function of the primary visual cortex may be a potentially useful marker for distinguishing LBD from AD.

\section{Frontotemporal lobar degeneration}

FTLD is the third most common type of degenerative dementia, after $\mathrm{AD}$ and $\mathrm{LBD},{ }^{25}$ and is the most common cause of dementia in people under 60 years of age. ${ }^{1}$ FTLD is a heterogeneous group of disorders characterized by disturbances of behavior and personality and by different types of language impairment, with or without concomitant features of motor neuron disease or parkinsonism. ${ }^{26}$ FTLD can be differentiated into three forms as follows: behavioral variant frontotemporal dementia, semantic dementia, and progressive nonfluent aphasia. ${ }^{9,25}$ Behavioral variant frontotemporal dementia is characterized by progressive behavioral impairments and a decline in executive function with frontal lobe-predominant atrophy. Semantic dementia is described by a loss of object knowledge with prominent anomia and asymmetrical atrophy of the anterior temporal lobes. Progressive nonfluent aphasia is characterized by expressive or motor speech deficits with predominantly left perisylvian atrophy. Detailed neuropathological studies have produced proteinopathies that relate to FTLD, such as inclusions of hyperphosphorylated microtubule-associated protein tau, transactive response DNA-binding protein TDP-43, fusedin-sarcoma, or as yet unidentified proteins in affected brain regions. Although not the case for the type of proteinopathy, the sites of neurodegeneration correlate relatively well with the clinical presentation of FTLD. ${ }^{26}$

Anatomically, FTLD is characterized by varying degrees of left and right frontal and temporal lobe atrophy. Patients with semantic dementia exhibit atrophy in the temporal pole, inferior temporal cortex, and fusiform gyrus, and this atrophy is usually much more prominent in the left hemisphere. ${ }^{27,28}$ Dysfunction of the fusiform gyrus may result in impaired reading and writing of words in patients with semantic dementia. The Japanese writing system is unique in that it is composed of two different orthographies, ie, Kanji (ideograms) and Kana (phonograms). ${ }^{29,30}$ Some case studies have described patients with semantic dementia who made errors while reading Kanji, but had a preserved ability to read Kana. ${ }^{31,32}$ Additionally, one study demonstrated that patients with semantic dementia exhibit poor performance when reading Hanja (ideograms) compared with AD patients. ${ }^{33}$ In contrast, FTLD patients (behavioral variant frontotemporal dementia and semantic dementia) with atrophy of the right temporal lobe show progressive prosopagnosia. ${ }^{34-37}$ Patients with behavioral variant frontotemporal dementia also exhibit severely impaired recognition of facial expressions for basic emotions, including happiness, sadness, disgust, fear, surprise, and anger. ${ }^{38-40}$ However, unlike patients with AD and LBD, those with FTLD tend to perform rather well in tests of visuospatial skills. ${ }^{4}$ This is consistent with the relative preservation of the occipitoparietal cortical regions. ${ }^{4}$ Thus, 
it is likely that visual impairments in FTLD patients are characterized by alexia (in particular, ideogram alexia) or prosopagnosia (including facial expression), thus reflecting dysfunction of the higher-level ventral stream.

\section{Mild cognitive impairment}

MCI refers to a mild regression of cognitive functions, specifically, memory impairment. The condition is not sufficiently severe to be classified as dementia because MCI patients retain abilities associated with the activities of daily living. ${ }^{41}$ MCI is a heterogeneous condition with a variety of clinical outcomes, the presence of which correlates with risk of AD as well as preclinical stages of other dementia subtypes. ${ }^{42}$ Indeed, a high percentage of MCI patients develop dementia within a few years. ${ }^{43}$ Petersen et $\mathrm{al}^{44}$ established a set of criteria to diagnose people suffering from MCI (subjective memory complaints, memory impairments, intact general cognitive functioning, preserved activities of daily living, and no diagnosis of dementia). However, people suffering from MCI vary considerably in terms of clinical symptoms and prognosis. Thus, the concept of MCI has been refined by distinguishing MCI subtypes according to the presence/ absence of episodic memory impairments (amnestic or nonamnestic) and the number of affected cognitive domains (single-domain or multiple-domain) ${ }^{43} \mathrm{MCI}$ has four clinical subtypes, ie, single-domain amnestic MCI (characterized by memory impairments only), multiple-domain amnestic MCI (characterized by memory impairments and impairments in additional cognitive domains), single-domain nonamnestic MCI (characterized by preserved memory but an overt decline in another cognitive domain), and multiple-domain nonamnestic MCI (characterized by preserved memory but with evidence of decline in several cognitive domains). ${ }^{41}$ Amnestic MCI is more likely to lead to AD compared with nonamnestic MCI, and the prognosis is even worse if amnestic decline is accompanied by impairments in other cognitive functions. In contrast, single-domain nonamnestic MCI may progress to LBD or FTLD, while multiple-domain nonamnestic MCI appears to lead to LBD. Therefore, it is important to detect and subtype MCI patients early on, to enable interventions that can help slow down the progression of the disease.

\section{Potential use of VEPs and ERPs in differential diagnosis of dementia Rationale for multimodal visual stimuli}

Two forms of VEP exist, ie, transient and steady-state.? Transient VEPs are obtained at low stimulus rates, while steady-state VEPs are elicited by repetitive frequent stimuli. ${ }^{7}$ Transient VEPs elicited by chromatic (red-green) sinusoidal gratings presented at low temporal frequencies with equal luminance and high spatial frequency are suitable for examining the lower levels of the parvocellular pathway within the primary visual cortex (Figure 2A). ${ }^{16,45,46}$ This type of stimulus evokes a characteristic negative wave (N120) with a peak latency around 120 milliseconds (Figure 2A). Conversely, steady-state VEPs elicited by achromatic (black-white) sinusoidal gratings presented at high temporal frequencies with low contrast and low spatial frequencies are useful for evaluating the magnocellular pathway within the primary visual cortex (Figure 2B). This stimulation induces a positive peak (P1) around 120 milliseconds followed by steady-state responses (Figure 2B). ${ }^{16,45,46}$

In terms of higher-level visual pathways, categoryspecific ERPs elicited by face, object, and word stimuli have been successfully used to evaluate the functional specialization of the ventral pathway. ${ }^{29,47,48}$ Faces, objects, and words (Kanji and Kana) evoke a negative occipitotemporal component with a peak latency around 170 milliseconds (N170, Figure 3). ${ }^{16,47}$ However, the N170 is right-lateralized for faces, is smaller and bilateral for objects, and is left-lateralized for words. ${ }^{16}$ Interestingly, Kanji (ideograms) and Kana (phonograms) were processed differently in healthy Japanese participants in our previous ERP study. ${ }^{29}$ Similarly, in our previous functional magnetic resonance imaging study, we found that the left inferior temporal region (Brodmann area 37) showed greater activation during a Kanji reading and we observed significant activation of the left inferior parietal lobule (Brodmann area 40) during the Kana reading in healthy Japanese participants. ${ }^{30}$ These findings suggest that an overlapping network was engaged while participants read Kanji and Kana, and that the left inferior temporal cortex was responsible for Kanji reading while the left inferior parietal lobule was responsible for Kana reading. ${ }^{30}$ Kanji and Kana contain a greater amount of high spatial frequency and low spatial frequency information, respectively. ${ }^{29,30}$ Therefore, the N170 component is useful for evaluating the ventral (particularly, the left inferior temporal cortex) pathway during Kanji reading, and useful for evaluating the dorsal (particularly, the left inferior parietal lobule) pathway during Kana reading.

Coherent motion stimuli using random dots, such as radial optic flow and horizontal motion (Figure 4A), can be useful for examining higher-level dorsal function (global motion processing). Radial optic flow motion is a type of complex visual motion related to self-motion perception. In contrast, 
A Chromatic stimulus

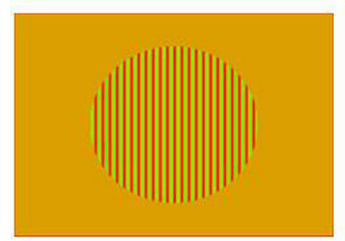

B Achromatic stimulus

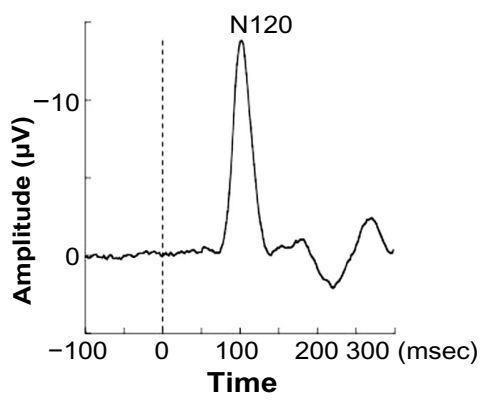

Waveforms

Steady-state responses
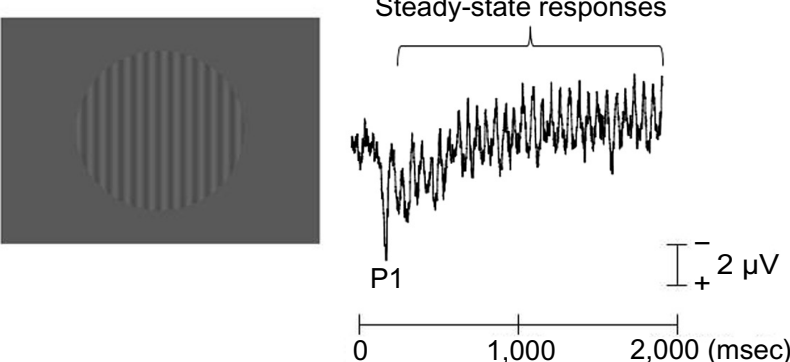

\section{FFTs}
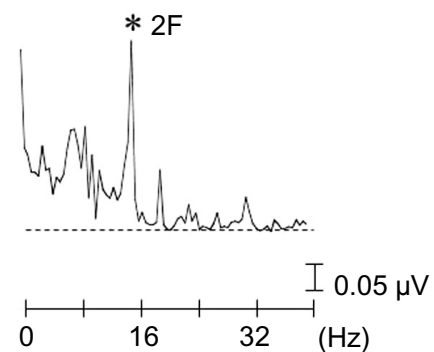

Figure 2 Visual evoked potentials elicited by chromatic and achromatic stimuli.

Notes: (A) Chromatic visual evoked potentials were elicited by red/green chromatic sinusoidal gratings with equal luminance (visual angle, $10 \times 10^{\circ}$; mean luminance, 21 cd/ $\mathrm{m}^{2}$; spatial frequency, two cycles per degree), presented for 200 milliseconds (msec). A negative component (NI20) was elicited in occipital regions (maximum at the Oz electrode). (B) Achromatic visual evoked potentials were elicited by achromatic (black/white) low-contrast (16.6\%) sinusoidal gratings (visual angle, I0×I0 ; mean luminance, $21 \mathrm{~cd} / \mathrm{m}^{2}$; spatial frequency, one cycle per degree), presented for $2,000 \mathrm{msec}$ and rapidly alternated in a square-wave fashion at $8 \mathrm{~Hz}(16 \mathrm{reversals}$ per second). A positive component at around $120 \mathrm{msec}(\mathrm{PI})$ and subsequent quasi-sinusoidal waveforms corresponding to the reversal frequency (I6 Hz) were elicited at the occipital regions (maximum at the Oz electrode). In the fast Fourier transforms (FFTs), the second harmonic (2F) was found to be a major component. Reprinted from J Alzheimers Dis. 20I2;3I Suppl 3. Yamasaki T, Horie S, Muranaka H, Kaseda Y, Mimori Y, Tobimatsu S. Relevance of in vivo neurophysiological biomarkers for mild cognitive impairment and Alzheimer's disease. Pages 137-154. Copyright 2012, with permission from IOS Press. ${ }^{16}$

\section{A Face stimulus}

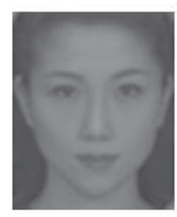

Neutral face

\section{B Word stimuli}

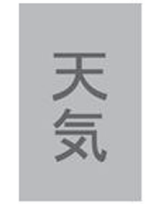

Kanji (ideograms)

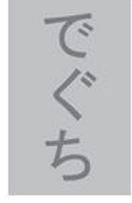

Kana (phonograms)
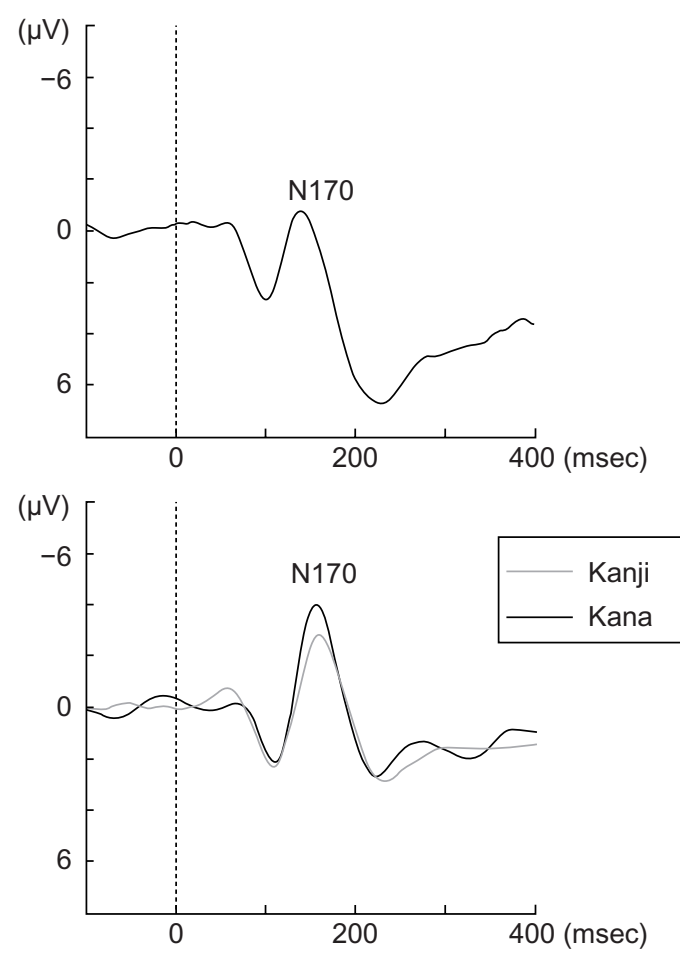

Figure 3 Event-related potentials elicited by face and word stimuli.

Notes: (A) For face stimuli, neutral faces were presented for 300 milliseconds (msec). A negative component (NI70) was elicited in bilateral occipitotemporal regions with right hemisphere dominance (maximum at the PO8 electrode). (B) For word stimuli, Kanji (ideogram) and Kana (phonogram) were randomly presented for 800 msec. A negative component ( $\mathrm{NI} 70$ ) was elicited by both stimuli in bilateral occipitotemporal regions with left hemisphere dominance (maximum at the PO7 electrode). Reprinted from J Alzheimers Dis. 20I2;3I Suppl 3. Yamasaki T, Horie S, Muranaka H, Kaseda Y, Mimori Y, Tobimatsu S. Relevance of in vivo neurophysiological biomarkers for mild cognitive impairment and Alzheimer's disease. Pages I37-I54. Copyright 20I2, with permission from IOS Press. ${ }^{16}$ 


\section{A Coherent motion stimuli}

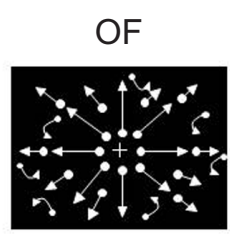

\section{B ERP waveforms}

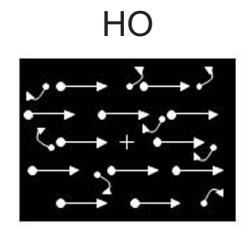

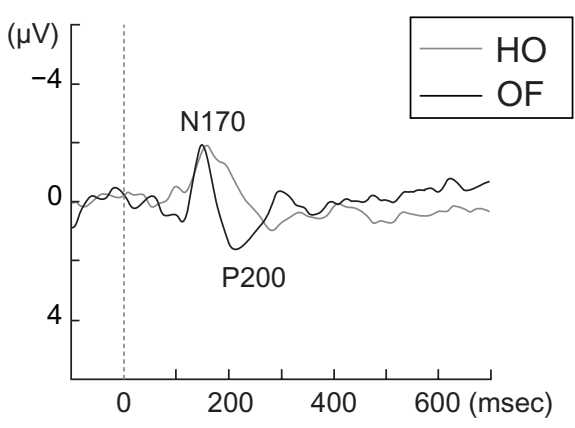

Figure 4 (A) Visual motion stimuli. Four hundred white dots (visual angle, $0.2 \times 0.2^{\circ}$; luminance, $48 \mathrm{~cd} / \mathrm{m}^{2}$ ) were randomly presented on a black background (visual angle, $50 \times 48^{\circ}$; luminance, $0.1 \mathrm{~cd} / \mathrm{m}^{2}$ ). The contrast level was $99.6 \%$. The white dots moved at a velocity of $5.0^{\circ} / \mathrm{second}$. When the white dots moved in an incoherent pattern, random stimulation was created. When the white dots moved in a coherent pattern, radial OF and HO motion were perceived. (B) Two major event-related potential components (NI70, P200) were obtained. The NI70 was evoked by both $\mathrm{HO}$ and OF stimuli while the P200 was only elicited by OF stimuli.

Note: Copyright (C) 2012 Takao Yamasaki et al. Adapted from Yamasaki T, Muranaka H, Kaseda Y, Mimori Y, Tobimatsu S. Understanding the pathophysiology of Alzheimer's disease and mild cognitive impairment: a mini review on fMRI and ERP studies. Neurol Res Int. 2012;20I2:719056.49

Abbreviations: OF, optic flow; $\mathrm{HO}$, horizontal motion; ERP, event related potential; msec, milliseconds.

horizontal motion refers to simple unidirectional motion. ${ }^{16,49}$ Our recent functional magnetic resonance imaging study revealed that the ventrodorsal stream (inferior parietal lobule) is important for optic flow motion processing, whereas the dorsodorsal stream (superior parietal lobule) is more closely involved in horizontal motion processing. ${ }^{16,49}$ Our ERP studies demonstrated that the perception of these stimuli was associated with two major components (N170 and P200) (Figure 4B). ${ }^{16,49-51}$ In terms of source analyses, the occipitotemporal N170 component appeared to originate from the V5/middle temporal area, and was evoked by both types of stimuli. In contrast, the parietal P200 component was found to originate in the inferior parietal lobule (BA 40), and was elicited only by optic flow stimuli. ${ }^{16,49-51}$

\section{Application of VEPs and ERPs with multimodal visual stimuli to $\mathrm{MCl}$}

Recently, we systematically examined parallel visual pathway function in patients with amnestic MCI (who later converted to $\mathrm{AD}$ ) using multimodal visual stimuli. ${ }^{16}$ Based on the behavioral characteristics of AD, we expected to find dysfunction of higher-level visual pathways (particularly, higher dorsal dysfunction) with preserved primary visual cortex function in amnestic MCI patients. As a measure of lower-level visual function (the primary visual cortex), we recorded the N120 component elicited by a chromatic stimulus and the steady-state responses evoked by an achromatic stimulus in MCI patients. We found these measures to be normal, indicating that amnestic MCI patients have normal lower-level parvocellular and magnocellular pathways.

In terms of higher-level function, we found normal ERPs elicited by faces and Kanji words in amnestic MCI patients, ${ }^{16}$ suggesting that the ventral stream was intact. When studying higher-level dorsal stream function, we found that the N170 component elicited by Kana words was significantly prolonged in amnestic MCI patients. ${ }^{16}$ This implies impaired dorsal function (inferior parietal lobule function) in MCI patients. To assess higher-level dorsal stream function, we also evaluated motion perception in amnestic MCI patients. ${ }^{16,49-51}$ We found no significant differences in the N170 components elicited by optic flow and horizontal motion stimuli between amnestic MCI patients and healthy elderly controls (Figure 5). However, we observed a significantly prolonged P200 latency for optic flow stimuli in amnestic MCI patients compared with healthy elderly controls (Figure 5). ${ }^{50,51}$ Therefore, within the dorsal stream, the ventrodorsal stream (inferior parietal lobule) related to optic flow perception but not the dorsodorsal stream (superior parietal lobule) is selectively impaired in amnestic MCI patients. ${ }^{49-51}$ Overall, our VEP and ERP studies indicate that patients with amnestic MCI are affected by selective impairment of the higher-level dorsal stream with preserved primary visual cortex function. Our electrophysiological findings were consistent with the behavioral characteristics of AD.

In terms of visual function in people with LBD, behavioral and neuroimaging findings indicate that these patients have impairments in both the lower-level and higher-level visual pathways. We conducted a preliminary examination of visual function in one nonamnestic MCI patient who developed dementia with Lewy bodies. This patient showed a prolonged N120 latency in response to chromatic stimuli and an ill-defined steady-state response to achromatic stimuli compared with individuals with amnestic MCI (who developed AD) and healthy elderly people. These 
A OF
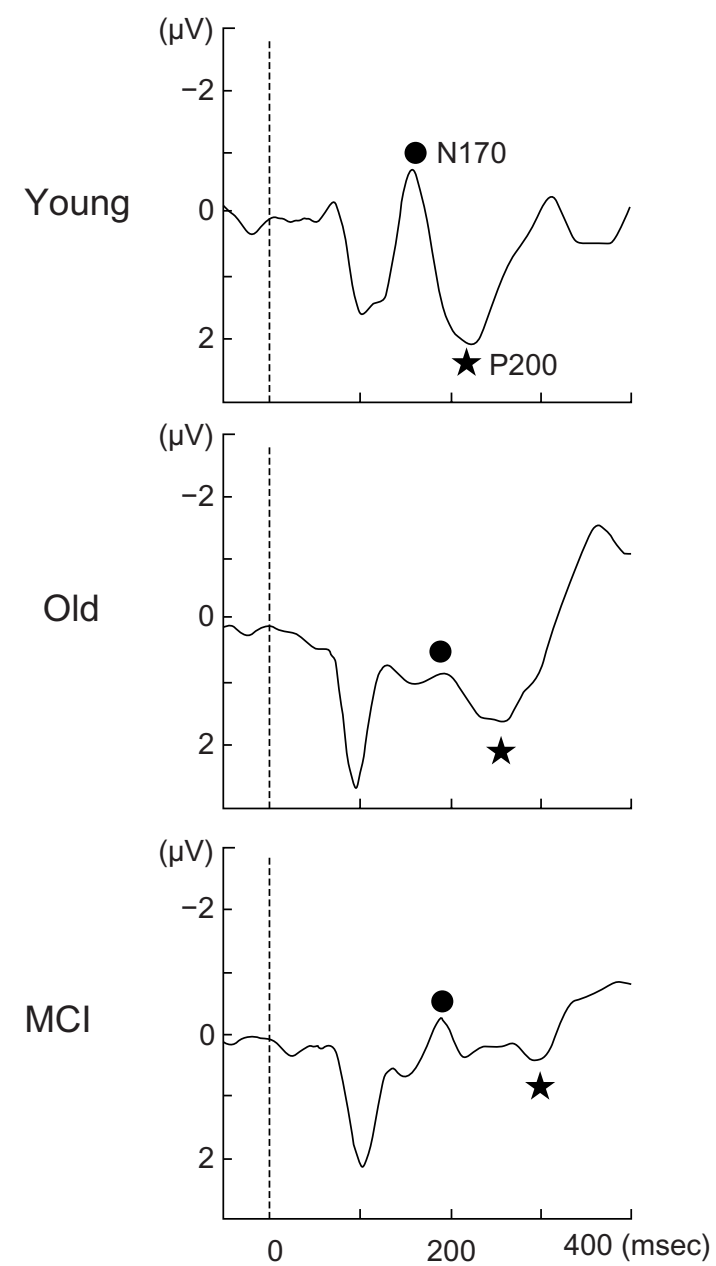

B $\mathrm{HO}$
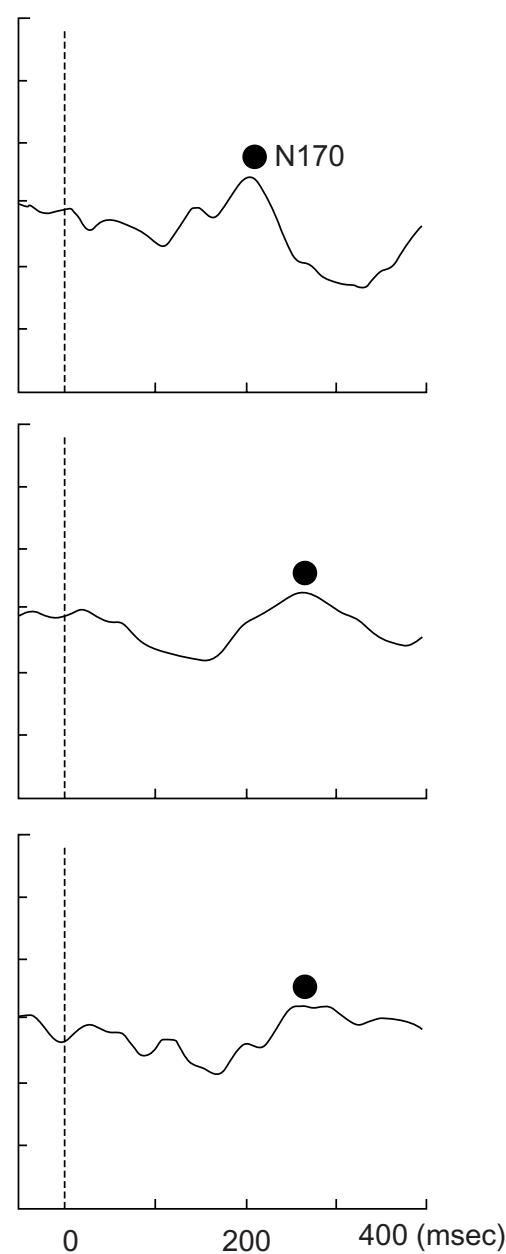

Figure 5 Grand-averaged event-related potentials at the right parietal electrode in response to OF (A) and $\mathrm{HO}$ (B) stimuli in each group.

Notes: There were no significant differences in the NI70 component elicited by the OF and $\mathrm{HO}$ stimuli between amnestic $\mathrm{MCl}$ patients and healthy elderly controls. In contrast, a significantly prolonged $\mathrm{P} 200$ latency was observed in response to OF stimuli in amnestic $\mathrm{MCl}$ patients compared with healthy elderly controls $(P<0.00 \mathrm{I})$.

(C) 2012 IEEE. Reprinted, with permission, from Yamasaki T, Goto Y, Ohyagi Y, et al. A deficit of dorsal stream function in patients with mild cognitive impairment and Alzheimer's disease. Presented at the 2012 IEEE/ICME International Conference on Complex Medical Engineering, July I-4, 20I2, Kobe, Japan. ${ }^{51}$

Abbreviations: OF, optic flow; $\mathrm{HO}$, horizontal motion; $\mathrm{MCl}$, mild cognitive impairment.

findings demonstrate impaired lower-level parvocellular and magnocellular pathways in a patient with nonamnestic MCI (dementia with Lewy bodies). This patient also exhibited a prolonged N170 component elicited by faces and words and prolonged N170 and P200 components in response to optic flow motion. These findings suggest that this patient was affected by impaired higher-level visual pathways. Therefore, our VEP and ERP findings are in accordance with neuroimaging reports of hypoperfusion or hypometabolism in the occipital lobes (primary visual cortex) and posterior parietotemporal lobes in patients suffering from dementia with Lewy bodies.

Although we have not yet tested patients with FTLD, we previously evaluated visual function in an individual with visual agnosia and prosopagnosia (without alexia) due to multiple sclerosis. This patient had selective occipitotemporal lesions involving the higher-level ventral pathway. ${ }^{47}$ Multimodal VEP and ERP testing demonstrated dysfunctional object and face perception with intact word perception in the ventral pathway. Additionally, higher-level dorsal pathway (motion perception) and lower-level primary visual cortex function were preserved. These findings are consistent with those of clinical and neuroimaging reports regarding lesions in the ventral pathway. ${ }^{47}$ Therefore, our VEP and ERP techniques may be able to detect selective impairment of the higher-level ventral pathway in patients with FTLD. However, further VEP and ERP studies with a large number of LBD and FTLD patients are necessary to confirm these results. 


\section{Ideal biomarkers in dementia}

Based on reports from research groups examining the biomarkers of $\mathrm{AD}$, the ideal biomarker for $\mathrm{AD}$ should detect a fundamental neuropathological feature, and be validated in neuropathologically confirmed cases. It should have a sensitivity of $>80 \%$ for detecting $\mathrm{AD}$, and a specificity of $>80 \%$ for distinguishing other dementias. It should also be reliable, reproducible, noninvasive, simple to perform, and inexpensive. ${ }^{52}$

Our VEP and ERP techniques can detect subtle changes in visual function in MCI patients. The findings of our studies are consistent with neuropsychological, neuroimaging, and neuropathological findings regarding degenerative dementia. Thus, our VEP and ERP techniques may be useful for detecting specific neuropathological markers in different types of degenerative dementia. Compared with genetic, biochemical, and neuroimaging methods, VEP and ERP measurements are cost-effective and noninvasive. Therefore, such electrophysiological techniques (perhaps coupled with other biomarkers) could be a useful approach for differentiating between AD and other major degenerative dementias, although future research is necessary to examine the sensitivity and specificity of our techniques.

\section{Conclusion}

The present review illustrates the ways in which three major types of neurodegenerative dementia, $\mathrm{AD}, \mathrm{LBD}$, and FTLD, exhibit specific patterns of visual dysfunction as early behavioral signs. Such visual impairments are manifestations of topographic patterns of cortical neuropathology, characteristic for each dementia type. AD is characterized by neuropathological changes in the posterior temporoparietal lobes with preserved occipital lobe (primary visual cortex) function. AD pathology may lead to abnormalities in higher-level ERPs (particularly, higher-level dorsal ERPs) with preserved lower-level VEPs. LBD is characterized by neuropathological changes in the occipital lobe (primary visual cortex) and the temporoparietal lobe. Thus, lowerlevel VEPs may be especially affected by LBD pathology, although higher-level ERPs are also affected. Individuals with FTLD exhibit neuropathological changes in the frontotemporal lobe, and not the posterior parietal lobe. This condition may induce abnormalities in higher-level ventral ERPs with normal lower-level VEPs and higher-level dorsal ERPs. Electrophysiological measures can detect subtle functional changes in the brain that are not detectable at a clinical or behavioral level. Therefore, our VEP and ERP techniques, which incorporate multimodal visual stimuli, have potential for detecting differential diagnostic biomarkers for major neurodegenerative dementias, especially for individuals in the early stage of dementia (such as MCI).

\section{Acknowledgments}

This study was supported in part by Grants-in-Aid for Scientists (18890131, 20591026) from the Ministry of Education, Culture, Sports, Science and Technology in Japan. We would like to thank Doctors Jun-ichi Kira, Yasumasa Ohyagi, Yoshinobu Goto, Akira Monji, Shigenobu Kanba, Shinji Munetsuna, Katsuya Ogata, Kensuke Sasaki, Motozumi Minohara, Katsuko Minohara, Shizuka Horie, Ikue Ijichi, Yuka Miyanaga, Chiharu Kurita, and Sachiko Takashima for their research assistance.

\section{Disclosure}

The authors report no conflicts of interest in this work.

\section{References}

1. Bird TD, Miller BL. Alzheimer's disease and other dementias. In: Hauser S, Josephson S, editors. Harrison's Neurology in Clinical Medicine. 2nd ed. London, UK: McGraw-Hill Medical; 2010.

2. Jellinger KA. Neuropathological aspects of Alzheimer disease, Parkinson disease and frontotemporal dementia. Neurodegener Dis. 2008;5:118-121.

3. Berti V, Pupi A, Mosconi L. PET/CT in diagnosis of dementia. Ann NYAcad Sci. 2011;1228:81-92

4. Karantzoulis S, Galvin JE. Distinguishing Alzheimer's disease from other major forms of dementia. Expert Rev Neurother. 2011;11: $1579-1591$.

5. Thies W, Bleiler L, Alzheimer's Association. 2013 Alzheimer's disease facts and figures. Alzheimers Dement. 2013;9:208-245.

6. Risacher SL, Saykin AJ. Neuroimaging and other biomarkers for Alzheimer's disease: the changing landscape of early detection. Annu Rev Clin Psychol. 2013;9:621-648.

7. Tobimatsu S, Celesia GG. Studies of human visual pathophysiology with visual evoked potentials. Clin Neurophysiol. 2006;117: 1414-1433.

8. Yamasaki T, Tobimatsu S. Electrophysiological assessment of the human visual system. In: Harris JM, Scott J, editors. Neuroscience Research Progress, Visual Cortex: Anatomy, Functions and Injuries. New York, NY, USA: Nova Science Publishers; 2012.

9. Possin KL. Visual spatial cognition in neurodegenerative disease. Neurocase. 2010;16:466-487.

10. Chang LY, Lowe J, Ardiles A, et al. Alzheimer's disease in the human eye. Clinical tests that identify ocular and visual information processing deficit as biomarkers. Alzheimers Dement. 2014;10:251-261.

11. Moreno-Ramos T, Benito-León J, Villarejo A, Bermejo-Pareja F. Retinal nerve fiber layer thinning in dementia associated with Parkinson's disease, dementia with Lewy bodies, and Alzheimer's disease. J Alzheimers Dis. 2013;34:659-664.

12. Ho WL, Leung Y, Tsang AW, So KF, Chiu K, Chang RC. Review: tauopathy in the retina and optic nerve: dose it shadow pathological changes in the brain? Mol Vis. 2012;18:2700-2710.

13. Livingstone M, Hubel D. Segregation of form, color, movement, and depth: anatomy, physiology, and perception. Science. 1988;240 740-749.

14. Braak H, Braak E. Neuropathological stageing of Alzheimer-related changes. Acta Neuropathol. 1991;82:239-259. 
15. Mendez MF, Mendez MA, Martin R, Smyth KA, Whitehouse PJ. Complex visual disturbances in Alzheimer's disease. Neurology. 1990;40:439-443.

16. Yamasaki T, Horie S, Muranaka H, Kaseda Y, Mimori Y, Tobimatsu S. Relevance of in vivo neurophysiological biomarkers for mild cognitive impairment and Alzheimer's disease. J Alzheimers Dis. 2012;31 Suppl 3:137-154.

17. Benson DF, Davis RJ, Snyder BD. Posterior cortical atrophy. Arch Neurol. 1988;45:789-793.

18. Nestor PJ, Caine D, Fryer TD, Clarke J, Hodges JR. The topography of metabolic deficits in posterior cortical atrophy (the visual variant of Alzheimer's disease) with FDG-PET. J Neurol Neurosurg Psychiatry. 2003; 74:1521-1529.

19. McKeith IG, Dickson DW, Lowe J, et al; Consortium on DLB. Diagnosis and management of dementia with Lewy bodies: third report of the DLB consortium. Neurology. 2005;65:1863-1872.

20. McKeith IG. Consensus guidelines for the clinical and pathologic diagnosis of dementia with Lewy bodies (DLB): report of the Consortium on DLB International Workshop. J Alzheimers Dis. 2006;9 Suppl 3: 417-423.

21. Calderon J, Perry RJ, Erzinclioglu SW, Berrios GE, Dening TR, Hodges JR. Perception, attention, and working memory are disproportionately impaired in dementia with Lewy bodies compared with Alzheimer's disease. J Neurol Neurosurg Psychiatry. 2001;70:157-164.

22. Mosimann UP, Mather G, Wesnes KA, O’Brien JT, Burn DJ, McKeith IG. Visual perception in Parkinson disease dementia and dementia with Lewy bodies. Neurology. 2004;63:2091-2096.

23. Minoshima S, Foster NL, Sima AA, Frey KA, Albin RL, Kuhl DE. Alzheimer's disease versus dementia with Lewy bodies: cerebral metabolic distinction with autopsy confirmation. Ann Neurol. 2001;50:358-365.

24. Lobotesis K, Fenwick JD, Phipps A, et al. Occipital hypoperfusion on SPECT in dementia with Lewy bodies but not AD. Neurology. 2001;56:643-649.

25. Mohandas E, Rajmohan V. Frontotemporal dementia; an updated overview. Indian J Psychiatry. 2009;51 Suppl 1:65-69.

26. Sieben A, Van Langenhove T, Engelborghs S, et al. The genetics and neuropathology of frontotemporal lobar degeneration. Acta Neuropathol. 2012;124:353-372.

27. Galton CJ, Patterson K, Graham K, et al. Differing patterns of temporal atrophy in Alzheimer's disease and semantic dementia. Neurology. 2001;57:216-225.

28. Chan D, Fox NC, Scahill RI, et al. Patterns of temporal lobe atrophy in semantic dementia and Alzheimer's disease. Ann Neurol. 2001;49: 433-442.

29. Horie S, Yamasaki T, Okamoto T, Nakashima T, Ogata K, Tobimatsu S. Differential roles of spatial frequency on reading processes for ideograms and phonograms: a high-density ERP study. Neurosci Res. 2012;72:68-78.

30. Horie S, Yamasaki T, Okamoto T, et al. Distinct role of spatial frequency in dissociative reading of ideograms and phonograms: an fMRI study. Neuroimage. 2012;63:979-988.

31. Fushimi T, Komori K, Ikeda M, Patterson K, Ijuin M, Tanabe H. Surface dyslexia in a Japanese patient with semantic dementia: evidence for similarity-based orthography-to phonology translation. Neuropsychologia. 2003;41:1644-1658.

32. Nakamura H, Nakanishi M, Hamanaka T, Nakaaki S, Yoshida S. Surface dyslexia and visual word recognition in Japanese. High Brain Funct Res. 2000;20:136-144.

33. Suh MK, Kim EJ, Lee BH, et al. Hanja (Ideogram) alexia and agraphia in patients with dementia. Neurocase. 2010;16:146-156.
34. Joubert S, Felician O, Barbeau E, et al. Impaired configurational processing in a case of progressive prosopagnosia associated with predominant right temporal atrophy. Brain. 2003;126:2537-2550.

35. George A, Jose J. Prosopagnosia: a rare presenting manifestation of frontotemporal lobar degeneration. Neurol India. 2009;57(1):58-60.

36. Nakachi R, Muramatsu T, Kato M, et al. Progressive prosopagnosia at a very early stage of frontotemporal lobar degeneration. Psychogeriatrics. 2007; 7:155-162.

37. Josephs KA, Whitwell JL, Vemuri P, et al. The anatomic correlate of prosopagnosia in semantic dementia. Neurology. 2008;71: $1628-1633$.

38. Rosen HJ, Pace-Savitsky K, Perry RJ, Kramer JH, Miller BL, Levenson RW. Recognition of emotion in the frontal and temporal variants of frontotemporal dementia. Dement Geriatr Cogn Disord. 2004; 17:277-281.

39. Diehl-Schmid J, Pohl C, Ruprecht C, Wagenpfeil S, Foerstl H, Kurz A. The Ekman 60 Faces Test as a diagnostic instrument in frontotemporal dementia. Arch Clin Neuropsychol. 2007;22:459-464.

40. Lough S, Kipps CM, Treise C, Watson P, Blair JR, Hodges JR. Social reasoning, emotion and empathy in frontotemporal dementia. Neuropsychologia. 2006;44:950-958.

41. Petersen RC, Doody R, Kurz A, et al. Current concepts in mild cognitive impairment. Arch Neurol. 2001;58:1985-1992.

42. Park KW, Kim EJ, Joo H, et al. Cognitive profiles and subtypes of patients with mild cognitive impairment: data from a clinical follow-up study. Int J Clin Med. 2012;3:352-360.

43. Petersen RC. Mild cognitive impairment as a diagnostic entity. J Intern Med. 2004;256:183-194.

44. Petersen RC, Smith GE, Waring SC, Ivnik RJ, Tangalos EG, Kokmen E. Mild cognitive impairment: clinical characterization and outcome. Arch Neurol. 1999;56:303-308.

45. Fujita T, Yamasaki T, Kamio Y, Hirose S, Tobimatsu S. Parvocellular pathway impairment in autism spectrum disorder: evidence from visual evoked potentials. Res Autism Spectr Disord. 2011;5:277-285.

46. Tobimatsu S, Tomoda H, Kato M. Parvocellular and magnocellular contributions to visual evoked potentials in humans: stimulation with chromatic and achromatic gratings and apparent motion. J Neurol Sci. 1995; 134:73-82.

47. Yamasaki T, Taniwaki T, Tobimatsu S, et al. Electrophysiological correlates of associative visual agnosia lesioned in the ventral pathway. J Neurol Sci. 2004;221:53-60.

48. Nakashima T, Goto Y, Abe T, et al. Electrophysiological evidence for sequential discrimination of positive and negative facial expressions. Clin Neurophysiol. 2008;119:1803-1811.

49. Yamasaki T, Muranaka H, Kaseda Y, Mimori Y, Tobimatsu S. Understanding the pathophysiology of Alzheimer's disease and mild cognitive impairment: a mini review on fMRI and ERP studies. Neurol Res Int. 2012;2012:719056.

50. Yamasaki T, Goto Y, Ohyagi Y, et al. Selective impairment of optic flow perception in amnestic mild cognitive impairment: evidence from event related potentials. J Alzheimers Dis. 2012;28:695-708.

51. Yamasaki T, Goto Y, Ohyagi Y, et al. A deficit of dorsal stream function in patients with mild cognitive impairment and Alzheimer's disease. Presented at the 2012 IEEE/ICME International Conference on Complex Medical Engineering, July 1-4, 2012, Kobe, Japan.

52. The Ronald Nancy Reagan Research Institute of the Alzheimer's Association the National Institute on Aging Working Group. Consensus report of the working group on: "molecular and biochemical markers of Alzheimer's disease". Neurobiol Aging. 1998;19:109-116. 
Current Biomarker Findings

\section{Publish your work in this journal}

Current Biomarker Findings is an international, peer-reviewed, open access journal publishing original research, reports, reviews and commentaries on all areas of biomarker research. The manuscript management system is completely online and includes a very quick and fair

peer-review system. Visit http://www.dovepress.com/testimonials.php to read real quotes from published authors. 